\title{
Viscoelasticity Cannot Create Stability
}

1. Delayed instabilities in viscoelastic solids through a metric description Authors: E. Urbach and E. Efrati arXiv:1711.09491

2. The metric description of viscoelasticity and instabilities in viscoelastic solids Authors: E. Urbach and E. Efrati arXiv:1806.01237

\section{Recommended with a Commentary by Sam Dillavou and Shmuel Rubinstein Harvard University, Cambridge MA, USA}

From colloidal gels [1] to tectonic faults [2], many systems exhibit the memory effect of delayed failure. In many systems, this phenomenon is clearly associated with stress corrosion induced by chemistry and other irreversible processes [3-4] . However, in some cases [1-2, 5] the source of the history-dependent failure is less clear and may stem form configurational and visco-elastic effects. A robust and flexible framework that describes the mechanics of viscoelasticity has yet to emerge, and often as a result, one resorts to computational models to describe these systems. In two recent pre-prints, Urbach and Efrati propose an intuitive geometrical description of viscoelasticity, in which a continually evolving metric tensor dictates the instantaneous local lowest energy state of a material. Within this geometrical framework, the question of the viscoelastic origin for delayed stability received a surprising but definitive answer.

Consider a simple viscoelastic system like a rubber band starting from a relaxed state. Stretch the band in a single direction and release it immediately, and it will have the same elastic rest length, $\mathrm{L}_{0}$, as before. Stretch it again in the same way, and the stress-strain response will be identical. Now stretch the band and hold it at constant length $\mathrm{L}$ for an extended time $\mathrm{t}$, and three things occur. First, the stress exerted by the band decreases over time. Second, when released, the band will snap back to an intermediate length $\mathrm{L}_{1}$, somewhere between $\mathrm{L}_{0}$ and L. Finally, under no stress, the band will slowly shrink until it reaches its original rest length $\mathrm{L}_{0}$. Urbach and Efrati describe this trio of effects in a framework of changing rest-lengths (metrics.) The lowest elastic energy state of the band, that is, the instantaneous state the band wants to snap to, is given by $\bar{L}(t)$. The evolution equation for $\bar{L}$ contains two terms, one pulling $\bar{L}$ towards the current length of the band, L, and one pulling towards the original rest length $L_{0}$. Conceptually, it is now an easy jump to a 3D continua, e.g. a block of memory foam, in which the evolving rest length is generalized by an evolving rest metric $\overline{g_{\imath j}}(t)$, that is 'the metric on which the body is locally

stress-free and stationary.' The strain tensor of the body is calculated as $\varepsilon_{i j}(t)=\frac{1}{2}\left(g_{i j}(t)-\right.$ 
$\left.\overline{g_{\imath \jmath}^{0}}\right)$, where $\overline{g_{l \jmath}^{0}}$ is the instantaneous rest metric, and $g_{i j}(t)$ is the current configuration of the system. Here too the rest metric evolves toward two values, $g_{i j}^{0}$ and $g_{i j}(t)$.

The tidiness of this framework shines through most in its treatment of stability. In one dimension, these co-evolutions of $\bar{L}$ towards both $L_{0}$ and $L$ mean that the equilibrium position for $\bar{L}$ will lie at some value $L_{0}<\bar{L}<L$. In two dimensions or above, the corollaries are less trivial; because of the two terms in the evolution equation, any equilibrium position for vector $\bar{L}$ must be colinear with $L$ and $L_{0}$. Urbach and Efrati show a surprising result of this rule for incompressible viscoelastic materials in the linear regime in any dimension; no permanently stable states for $L$ may be created through the evolution of the instantaneous rest length $\bar{L}$, and conversely, a stable state when the system is relaxed will never lose stability due to viscoelasticity. As a demonstration of this principle, Urbach and Efrati utilize a model system: conic silicone-rubber shells of varying thicknesses. The thinnest shells are bi-stable - they hold their shape when inverted inside-out. In contrast to the thickest shells, which immediately snap back, inverting to their original shape. Shells of medium thickness snap back immediately when inverted, unless held in their inverted state for a period before release. That is, bi-stability is created through evolution of the system's rest metric. However, none of these created bi-stable states is permanent, although stability lasts for minutes if held long enough. This is the crux of the matter: viscoelasticity can delay the instability, but it cannot eliminate it.

The framework presented by Urbach and Efrati is mathematically elegant and clean and is thus a fitting tool to determine what general behaviors visco-elastic systems are capable of. For example, is there a point in the tectonic cycle past which an earthquake is inevitable, but the system is temporarily stable? The framework may also provide a backbone for the description of many complex systems of current interest. Several systems that have long been known to demonstrate viscoelastic behavior, such as frictional interfaces [6-7] and crumpled paper [8], have recently been shown to display a more complex memory than previously thought [9-10]. The capability of these systems, for example, to produce a non-monotonic evolution in stress under constant strain is also possible in the framework discussed here. Time will tell what complex and interesting systems this framework can describe.

\section{References}

[1] SB Lindstrom et. al. Structures, stresses, and fluctuations in the delayed failure of colloidal gels. Soft Matter (2012)

[2] Omori, F. On the after-shocks of earthquakes (Vol. 7). Imperial University of Tokyo. (1894)

[3] Wiederhorn, S.M. and Bolz, L.H. Stress corrosion and static fatigue of glass. Journal of the American Ceramic Society, 53(10), pp.543-548. (1970)

[4] Johnson, H.H., Morlet, J.G. and Troiano, A.R. Hydrogen, crack initiation, and delayed failure in steel. Trans. Met. Soc. AIME, 212. (1958)

[5] Freed, A.M. and Lin, J. Delayed triggering of the 1999 Hector Mine earthquake by viscoelastic stress transfer. Nature. (2001)

[6] E Rabinowicz, Friction and Wear of Materials Wiley New York (1965)

[7] JH Dieterich and BD Kilgore, Direct Observation of Frictional Contacts, Pure Appl Geophys (1994) 
[8] K Matan et. al., Crumpling a Thin Sheet. Phys Rev Let (2002)

[9] S Dillavou and SM Rubinstein, Nonmonotonic Aging and Memory in a Frictional Interface. Phys Rev Let (2018)

[10] Y Lahini et. al., Nonmonotonic Aging and Memory Retention in Disordered Mechanical Systems. Phys Rev Let (2017) 